\title{
COMMUTATIVE IDEMPOTENT RESIDUATED LATTICES
}

\author{
DAVID STANOVSKÝ
}

\begin{abstract}
We investigate the variety of residuated lattices with a commutative and idempotent monoid reduct.
\end{abstract}

A residuated lattice is an algebra $\mathbf{A}=(A, \vee, \wedge, \cdot, e, /, \backslash)$ such that $(A, \vee, \wedge)$ is a lattice, $(A, \cdot, e)$ is a monoid and for every $a, b, c \in A$

$$
a b \leq c \quad \Leftrightarrow \quad a \leq c / b \quad \Leftrightarrow \quad b \leq a \backslash c .
$$

The last condition is equivalent to the fact that $(A, \vee, \wedge, \cdot, e)$ is a lattice-ordered monoid and for every $a, b \in A$ there is a greatest $c$ such that $c b \leq a$ (denoted $a / b$ ) and a greatest $d$ such that $b d \leq a$ (denoted $b \backslash a)$. It is easy to see that the class $\mathcal{R} \mathcal{L}$ of all residuated lattices is a variety. We are concerned about the variety $\mathcal{C} \mathcal{I} d \mathcal{R} \mathcal{L}$ of commutative idempotent $(\mathrm{CI})$ residuated lattices, i.e. the subvariety of $\mathcal{R} \mathcal{L}$ given by equations

$$
x y \approx y x \quad \text { and } \quad x x \approx x .
$$

In other words, residuated lattices whose semigroup reduct is a semilattice. For example, every Heyting algebra is a CI residuated lattice, where $a b=a \wedge b$ and $a / b=b \backslash a=b \rightarrow a$ for every $a, b$ (see e.g. [3], p. 30).

Foundation of the theory of residuated lattices goes as far as to 1930's, when Dilworth and Ward [5] studied lattices of ring ideals. A recent introduction can be found in [4] and [10] and commutative residuated lattices were particularly studied in [9]. We will use the notation and terminology of these papers. We also assume a basic familiarity with universal algebra, standard references are [3] and [12].

In CI residuated lattices, we drop the operation $\backslash$, since under commutativity $x / y \approx y \backslash x$. The lattice order will be denoted by $\leq$. We put $a \preceq b$ iff $a b=a$; hence $\preceq$ is the semilattice order, where - is regarded as the meet; $e$ is its top element. When refering to an order, we mean the lattice order $\leq$, unless explicitly stated otherwise. We put $A^{+}=\{a \in A: a \geq e\}$ and $A^{-}=\{a \in A: a \leq e\}$ and we call $\mathbf{A}^{+}$the positive cone and $\mathbf{A}^{-}$the negative cone of $\mathbf{A}$ (regarded as lattice-ordered monoids; indeed, they may not be closed on residuation).

The bottom element (in the lattice order) is denoted 0 and the top element is denoted 1 , if they exist; it is easy to see that, in any residuated lattice, if 0 exists, then 1 exists, $0 a=a 0=0$ and $a / 0=1 / a=1$ (see also [4]); particularly, 0 is also the bottom element of the semilattice order in any CI residuated lattice.

1991 Mathematics Subject Classification. 06F05.

Key words and phrases. residuated lattice, semilattice, finitely based variety, minimal variety.

The work is a part of the research project MSM 0021620839 financed by MŠMT. A partial support by the Grant Agency of the Czech Republic, grants \#201/02/0594 and \#201/02/0148 is acknowledged. 


\section{Motivation}

Our interest in this particular variety comes from the following observation.

1.1. Observation. Let $\mathcal{V}$ be a non-trivial subvariety of residuated lattices based (relatively to $\mathcal{R} \mathcal{L}$ ) by equations in the language of monoids. Then $\mathcal{V}$ contains $\mathcal{C I d \mathcal { R }} \mathcal{L}$ as a subvariety. (In other words, any monoid equation with a non-trivial residuated lattice model is implied by commutativity and idempotency.)

Proof. Let $u \approx v$ be an equation in the language of monoids valid in $\mathcal{V}$. In order to prove that every CI residuated lattice is in $\mathcal{V}$, it is enough to show that $u \approx v$ holds in every semilattice. Indeed, this happens, iff the terms $u$ and $v$ contain the same variables. Hence, suppose that a variable $x$ occurs in the term $u$ and does not occur in the term $v$. Put all other variables equal to $e$ and obtain an equation $x^{n} \approx e$ for some $n$, valid in $\mathcal{V}$. However, this implies that $\mathcal{V}$ is trivial, because any non-trivial lattice-ordered monoid contains an element $a$ comparable to $e$ and we get a contradiction either by $e<a \leq a^{2} \leq \cdots \leq a^{n}=e$ if $a>e$, or similarly if $a<e$.

Our motivation was the following result of Bahls, Cole, Galatos, Jipsen and Tsinakis [1].

1.2. Theorem. Let $\mathcal{V}$ be a non-trivial subvariety of residuated lattices based (relatively to $\mathcal{R} \mathcal{L}$ ) by equations in the language of lattices. Then $\mathcal{V}$ does not satisfy any non-trivial monoid equation (precisely, for every equation $\varepsilon$ in the language $\cdot, e$, if $\mathcal{V} \vDash \varepsilon$, then all monoids satisfy $\varepsilon$ ).

Proof. Let $\mathbf{L}$ be a bounded lattice. We construct a residuated lattice $\mathbf{L}^{\prime}$, whose monoid reduct is the free monoid over the alphabet $L$ and whose lattice reduct satisfies the same lattice equations as $\mathbf{L}$ (it generates the same variety as $\mathbf{L}$ ). We identify words of length $n$ over $L$ with $n$-tuples of elements of $L$ and define a lattice structure on the free monoid to be the ordinal sum of $\mathbf{L}^{0}$ (consisting of the empty word), $\mathbf{L}^{1}, \mathbf{L}^{2}, \mathbf{L}^{3}, \ldots$ (with the empty word on top). One can check that the resulting structure becomes a residuated lattice. Now, if a monoid identity holds in $\mathcal{V}$, it holds in $\mathbf{L}^{\prime}$ for every $\mathbf{L}$ satisfying the relative base of $\mathcal{V}$. Hence it holds in free monoids and thus in every monoid. See [1] for details.

Is there a similar theorem, with the role of lattice and monoid reducts interchanged?

1.3. Theorem. The variety $\mathcal{C I} d \mathcal{R} \mathcal{L}$ does not satisfy any non-trivial lattice equation (precisely, for every equation $\varepsilon$ in the language $\vee, \wedge$, if $\mathcal{C} \mathcal{I} d \mathcal{R} \mathcal{L} \vDash \varepsilon$, then all lattices satisfy $\varepsilon)$.

Proof. Let $\mathbf{L}$ be a bounded lattice. We construct a CI residuated lattice $\mathbf{L}^{\prime}$, whose lattice reduct satisfies the same lattice equations as $\mathbf{L}$ (it generates the same variety as $\mathbf{L}$ ). Let us denote 1 the top element of $\mathbf{L}$ and $e$ the bottom element of $\mathbf{L}$. Let $L^{\prime}$ be the disjoint union of $L$ and $\{0\}$. The lattice structure on $L^{\prime}$ is defined so that 0 is added to $\mathbf{L}$ as a new bottom element. We define the multiplication by $00=0 a=a 0=0$ for every $a \in L$ and $a b=a \vee b$ for every $a, b \in L$. It is easy to check that this is a lattice-ordered CI monoid and it admits residuation as follows: $a / 0=1,0 / a=0, a / b=a$ for $b \leq a$ and $a / b=0$ for $b \not \leq a, a, b \in L$. Now, if a lattice identity holds in $\mathcal{C} \mathcal{I} d \mathcal{R} \mathcal{L}$, it holds in $\mathbf{L}^{\prime}$ for every bounded lattice $\mathbf{L}$ and thus it holds in all lattices. 
1.4. Corollary. Let $\mathcal{V}$ be a non-trivial subvariety of residuated lattices based (relatively to $\mathcal{R} \mathcal{L}$ ) by equations in the language of monoids. Then $\mathcal{V}$ does not satisfy any non-trivial lattice equation.

Proof. According to Observation 1.1, the variety $\mathcal{C} \mathcal{I} d \mathcal{R} \mathcal{L}$ is a subvariety of $\mathcal{V}$ and thus Theorem 1.3 applies.

\section{BASIC PROPERTIES}

2.1. Lemma. Let $\mathbf{A}$ be a lattice-ordered idempotent monoid and $a, b \in A$.

(1) $a \wedge b \leq a b \leq a \vee b$.

(2) If $a, b \geq e$, then $a b=a \vee b$.

(3) If $a, b \leq e$, then $a b=a \wedge b$.

(4) If $a \leq e \leq a b$, then $a b=b$.

(5) If $a b \leq e \leq a$, then $a b=b$.

Proof. (1) $a \wedge b \leq a, b \leq a \vee b$, hence $a \wedge b=(a \wedge b)(a \wedge b) \leq a b \leq(a \vee b)(a \vee b)=a \vee b$.

(2) If $a \geq e$, then $a b \geq e b=b$ and similarly also $a b \geq a$. Thus $a b \geq a \vee b$. The other inequality was proven in (1). Similarly for (3).

(4) $b=e b \leq a b b=a b \leq e b=b$. Similarly for (5).

The following two statements about congruence lattices of CI residuated lattices are immediate consequences of results in [4] and [9]. The second sentence of Proposition 2.2 appears also in [8] (in a more general setting).

2.2. Proposition. The congruence lattice of $\mathbf{A}$ is isomorphic to the lattice of filters on $\mathbf{A}^{-}$. In particular, if $A$ is finite, then $\mathbf{C o n}(\mathbf{A}) \simeq\left(\mathbf{A}^{-}\right)^{\partial}$.

Proof. Blount and Tsinakis described in [4] a correspondence between congruences of a residuated lattice $\mathbf{A}$ and convex normal submonoids of $\mathbf{A}^{-}$. We prove that convex normal submonoids in CI residuated lattices are precisely filters.

Let $M \subseteq A^{-}$. Since $a \wedge b=a b$ for all $a, b \leq e, M$ is closed on meet iff it is closed on multiplication. If $e \in M$ (it indeed is, whenever $\mathbf{M}$ is a submonoid or a filter), then $M$ is convex iff it is an upper set. Hence, it remains to show that every filter is normal. Since $(b a) / b=(a b) / b \geq a$ for all $a, b$, every conjugation mapping $\gamma(x)=((b x) / b) \wedge e$ maps a negative element onto a greater one. Consequently, congruences of a CI resuduated lattice correspond to filters.

2.3. Corollary. A CI residuated lattice $\mathbf{A}$ is simple, iff $\left|A^{-}\right|=2$. It is subdirectly irreducible, iff e is completely join-irreducible.

It is well-known that residuated lattices are congruence distributive and congruence permutable. In particular, the negative cone of a non-trivial CI residuated lattice is always distributive (in fact, it is a Heyting algebra) and contains at least two elements.

\section{Finitely AND NON-Finitely BASEd SUbVARIETIES}

3.1. Proposition. CI residuated lattices have definable principle congruences.

Proof. Principal congruences correspond to principal filters, which are, of course, first-order definable. It can be checked easily that a congruence corresponding to a definable convex normal submonoid is also definable (generally for residuated lattices). 
In fact, N. Galatos proved a stronger result in [8]: principal congruences in commutative $n$-potent residuated lattices are equationally definable. This result is indeed more complicated.

3.2. Corollary. A subvariety $\mathcal{V}$ of $\mathcal{C I} d \mathcal{R} \mathcal{L}$ is finitely based, iff the class of subdirectly irreducible algebras in $\mathcal{V}$ is first-order definable.

Proof. This is an immediate consequence of a theorem of K. Baker and J. Wang $[2]$.

A non-finitely based variety of lattices was found by R. McKenzie in [11]. He constructed an infinite independent family $\varepsilon_{1}, \varepsilon_{2}, \ldots$ of lattice equations and finite lattices $\mathbf{B}_{1}, \mathbf{B}_{2}, \ldots$ such that $\mathbf{B}_{n} \not \models \varepsilon_{n}$ and $\mathbf{B}_{n} \vDash \varepsilon_{m}$ for every $m \neq n$. We modify his construction to get an example of a non-finitely based subvariety of CI residuated lattices.

3.3. Proposition. Let $\mathcal{V}$ be a variety with a lattice reduct and assume that for every finite lattice $\mathbf{L}$ there is an algebra $\mathbf{A}_{\mathbf{L}} \in \mathcal{V}$ such that $\mathbf{L}$ and $\left(A_{\mathbf{L}}, \vee, \wedge\right)$ satisfy the same lattice equations. Then the subvariety of $\mathcal{V}$ based (relatively to $\mathcal{V}$ ) by $\varepsilon_{1}, \varepsilon_{2}, \ldots$ is not finitely based.

Proof. Let us denote the subvariety $\mathcal{W}$. If there were a finite base $\Sigma$ of $\mathcal{W}$, by the compactness theorem, only finitely many $\varepsilon_{i}$ 's were necessary to prove that $\Sigma$ holds in $\mathcal{W}$. Thus there is $n$ such that $\mathcal{C} \mathcal{I} d \mathcal{R} \mathcal{L}, \varepsilon_{1}, \ldots, \varepsilon_{n} \vDash \Sigma$. Hence, since $\Sigma$ is a base of $\mathcal{W}$, a CI residuated lattice is in $\mathcal{W}$, iff it satisfies $\varepsilon_{1}, \ldots, \varepsilon_{n}$. But it means that $\mathbf{A}_{\mathbf{B}_{m+1}} \in \mathcal{W}$, because $\mathbf{B}_{m+1}$ satisfies all equations $\varepsilon_{1}, \ldots, \varepsilon_{m}$. On the other hand, $\mathcal{W} \vDash \varepsilon_{m+1}$ and $\mathbf{A}_{\mathbf{B}_{m+1}} \not \models \varepsilon_{m+1}$. This is a contradiction.

Proposition 3.3 applies to the variety $\mathcal{C} \mathcal{I} d \mathcal{R} \mathcal{L}$; we can take, for example, $\mathbf{A}_{\mathbf{L}}=$ $\mathbf{L}^{\prime}$ from the proof of Theorem 1.3. It applies also to the variety of cancellative residuated lattices, if we take $\mathbf{A}_{\mathbf{L}}=\mathbf{L}^{\prime}$ from the proof of Theorem 1.2.

\section{More EXAMPLES}

A complete lattice $\mathbf{L}$ is called infinitely join distributive, if $\bigvee_{x \in X}(x \wedge y)=$ $\left(\bigvee_{x \in X} x\right) \wedge y$ holds for any $X \subseteq L$ and $y \in L$.

Example. Let $\mathbf{D}$ be a complete infinitely join distributive lattice. Then the algebra $(D, \vee, \wedge, \wedge, 1, /)$ is a CI residuated lattice, where $a / b=\bigvee\{c: c \wedge b \leq a\}$. (Indeed, since $a / b$ is the greatest $c$ such that $c \wedge b \leq a$, it must be $\bigvee\{c: c \wedge b \leq a\}$. And the big join is less than $a$, if $\mathbf{D}$ is infinitely join distributive.)

Example. Let $\mathbf{L}$ be a bounded lattice and $\mathbf{D}$ a complete infinitely join distributive lattice, suppose $L \cap D=\emptyset$. We construct a CI residuated lattice $\mathbf{L} \sqcup \mathbf{D}$ on the set $L \cup D$. Let $\mathbf{L}, \mathbf{D}$ be sublattices of $\mathbf{L} \sqcup \mathbf{D}$ with all elements of $L$ greater then any element of $D$. Denote $e$ the bottom element of $\mathbf{L}$ and $t$ the top element of $\mathbf{D}$, while 0,1 refer to the top and bottom of $\mathbf{L} \sqcup \mathbf{D}$. Put $a b=a \vee b$ for $a, b \in L, a b=a \wedge b$ for $a, b \in D$ and $a b=b a=b$ for $a \in L, b \in D$. It is easy to check that this is a lattice-ordered CI monoid and it admits residuation as follows:

- $a / b=a$ for $e \leq b \leq a$.

- $a / b=1$ for $b \leq a, b \leq e$.

- $a / b=a$ for $a \leq e \leq b$.

- $a / b=t$ for $b \not \leq a, a, b \geq e$. 
- $a / b=\bigvee\{c \in D: c \wedge b \leq a\}$ for $b \not \leq a, a, b \leq e$.

Consequently, for every bounded lattice $\mathbf{L}$ and complete infinitely join distributive lattice $\mathbf{D}$, there is a $\mathrm{CI}$ residuated lattice $\mathbf{A}$ with $\left(A^{+}, \vee, \wedge\right)=\mathbf{L},\left(A^{-}, \vee, \wedge\right)=$ $\mathbf{D}+\{e\}$ and all elements comparable to $e$. Note that the lattice $\mathbf{L} \sqcup \mathbf{D}$ is subdirectly irreducible.

In particular, there exists a simple CI residuated lattice $\mathbf{L}^{\prime}$ with $\left(L^{\prime+}, \vee, \wedge\right)=$ L (take $\mathbf{D}$ trivial). By Lemma 2.1(2), any simple CI residuated lattice with no elements incomparable to the unit is some $\mathbf{L}^{\prime}$. Also, by Jónsson's lemma, $\mathbf{L}^{\prime}$ 's are the only subdirectly irreducible algebras in the variety they generate, hence they generate a proper subvariety of $\mathcal{C} \mathcal{I} d \mathcal{R} \mathcal{L}$. This variety is finitely based, according to Corollary 3.2. In fact, one can use the Galatos' algorithm [7] and find a basis: it is based (relatively to $\mathcal{C} \mathcal{I} d \mathcal{R} \mathcal{L})$ by the single equation $((e / x) \wedge e) \vee((y / x) \wedge e) \approx e$.

It is easy to check that there is (up to isomorphism) one 2-element CIRL, two 3-element CIRLs and four 4-element CIRLs. Using a computer, on can compute that there are twenty 5-element CIRLs; every 5-element lattice is a reduct of a CIRL; and in any 5-element lattice, one can choose $e \neq 0,1$ arbitrarily, except for the following case:

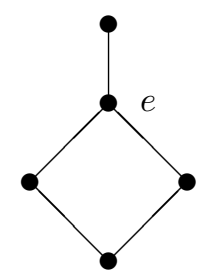

We proved that every bounded lattice is a subreduct of a CI residuated lattice. However, there is a 6-element lattice, which is not a reduct of a CI residuated lattice.

4.1. Proposition. Let $\mathbf{L}$ be a lattice and $\mathbf{M}_{n}$ be the $(n+2)$-element lattice with $n$ atoms, $n \geq 3$. Then the ordinal sum $\mathbf{L}^{\prime}$ of $\mathbf{L}$ and $\mathbf{M}_{n}$ (with $\mathbf{L}$ on top) is not a reduct of a CI residuated lattice.

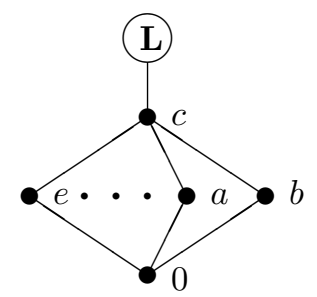

Proof. Assume there is a CI residuated lattice $\mathbf{A}$ with the lattice reduct $\mathbf{L}^{\prime}$. First of all, note that the unit element must be one of the atoms - otherwise, $\mathbf{A}^{-}$is not a non-trivial distributive lattice. Let us denote $e, a, b$ three distinct atoms and assume that $e$ is the unit element. Let $c=e \vee a \vee b$ be the top element of $\mathbf{M}_{n}$. It is well known (see [4]) and easy to prove that in any residuated lattice multiplication distributes over joins, in symbols

$$
x(y \vee z) \approx(x y) \vee(x z) .
$$


Using this identity, we get for every atom $x \neq e$ in $\mathbf{L}^{\prime}$ that $x c=x(e \vee x)=x \vee x=x$. Another use of this identity yields $a=a c=a(e \vee b)=a \vee(a b)$ and similarly $b=b \vee(a b)$, so $a b \leq a$ and $a b \leq b$ and thus $a b=0$. Now, choose $d \in L$. We have $(d a) \vee(d b)=d(a \vee b)=d c=d$ (because multiplication coincides with the join on positive elements). Hence, at least one of $d a, d b$ must be greater than $c$; assume it is $d a$. Then $c(d b) \leq(d a)(d b)=d(a b)=d 0=0$. However, this is possible iff $d b=0$, because $c x \geq c$ for every $x$ positive and we proved above that $c x=x$ for every atom $x \neq e$. But $d b \geq e b=b$, a contradiction.

A different argument shows examples of infinite lattices which are not reducts of any CI residuated lattice. Let $\mathbf{L}$ be an arbitrary simple atomless lattice (e.g. the dual of the lattice of subspaces of an infinite-dimensional vector space) and let $\mathbf{A}$ be a CI residuated lattice with the lattice reduct $\mathbf{L}$. By adding operations to a simple algebra, one gets again a simple algebra. Hence $\mathbf{A}$ is simple, but $\mathbf{A}^{-}$cannot have two elements, because there are no atoms in $\mathbf{A}$, which contradicts Corollary 2.3 .

The following propositions describe all totally ordered CI residuated lattices (i.e. those, where the lattice reduct is a chain).

4.2. Proposition. Let $\mathbf{A}=(A, \vee, \wedge, \cdot, e)$ be a structure such that $(A, \vee, \wedge)$ is a chain and $(A, \cdot, e)$ is a semilattice with a unit. Then the following are equivalent.

(1) A is a lattice-ordered monoid.

(2) $a b=a \vee b$ for every $a, b \in A^{+}, a b=a \wedge b$ for every $a, b \in A^{-}$and the semilattice reduct is a chain.

Proof. $(1) \Rightarrow(2)$ follows from Lemma 2.1. If $a, b$ are both positive or both negative, $2.1(2)$ or $2.1(3)$ applies. Otherwise, since $\leq$ is a chain, we may assume that $a \leq$ $e \leq b$. In this case, either $e \leq a b$ and 2.1(4) applies, or $a b \leq e$ and 2.1(5) applies.

$(2) \Rightarrow(1)$. Note that on the positive cone, $a \leq b$ iff $b \preceq a$, and on the negative cone, $a \leq b$ iff $a \preceq b$. Let $a \leq b$. We need to prove that $a c \leq b c$ for every $c \in A$. Since $(A, \preceq)$ is a chain, $a c \in\{a, c\}$ and $b c \in\{b, c\}$. Hence the only bad situation is either (a) $a c=a, b c=c$ and $a>c$, or (b) $a c=c, b c=b$ and $c>b$. We prove that none of them is actually possible. In (a), we have $c<a<b$ and $a \prec c \prec b$. The element $a$ can't be positive, because in this case $b$ is also positive and $a<b$ implies $b \prec a$. On the other hand, $a$ can't be negative, because then $c$ is also negative and $c<a$ implies $c \prec a$. This is a contradiction. In (b), we have $a<b<c$ and $b \prec c \prec a$ and a similar argument works.

4.3. Corollary. Let $\mathbf{A}=(A, \vee, \wedge, \cdot, e)$ be a structure such that $(A, \vee, \wedge)$ is a chain and $(A, \cdot, e)$ is a semilattice with a unit. Then the following are equivalent.

(1) $(A, \vee, \wedge, \cdot, e, /)$ is a residuated lattice for some /.

(2) $a b=a \vee b$ for every $a, b \in A^{+}, a b=a \wedge b$ for every $a, b \in A^{-}$, the semilattice reduct is a chain and for every $a, b$ there is the greatest $c$ such that $a c \leq b$.

In particular, for $A$ finite, the conditions are equivalent to

(3) $a b=a \vee b$ for every $a, b \geq e, a b=a \wedge b$ for every $a, b \leq e$ and the semilattice reduct is a chain with 0 in bottom.

Proof. (1) $\Leftrightarrow$ (2) follows obviously from the previous proposition. If (1),(2) are true, then (3) follows from the fact that 0 exists and $0 a=a 0=0$ for all $a$ in any residuated lattice with 0 . And if (3) holds, then there is always some $c$, namely 
$c=0$, such that $a c \leq b$, and thus there is also the greatest such $c$. (Note that it is enough to assume that the dual of $(A, \vee, \wedge)$ is well-ordered with a top element, not necessarily finite.)

\section{Minimal VARIETIES}

Minimal subvarieties of residuated lattices were investigated by several authors, particularly by N. Galatos in [6]. He found also minimal subvarieties of $\mathcal{C} \mathcal{I} d \mathcal{R} \mathcal{L}-$ they are just two. We shortly reprove his result.

A residuated lattice is called integral, if all its elements are negative. Let $\mathbf{C}_{2}$ be the two-element CI residuated lattice, $C_{2}=\{0,1\}, e=1$. Let $\mathbf{C}_{3}$ be the threeelement non-integral CI residuated lattice, $C_{3}=\{0, e, 1\}, 0<e<1$. (Note that, in fact, $\mathbf{C}_{2}$ is the only two-element residuated lattice and $\mathbf{C}_{3}$ is the only non-integral three-element residuated lattice.) Let $\mathcal{V}_{2}, \mathcal{V}_{3}$ be the varieties generated by $\mathbf{C}_{2}, \mathbf{C}_{3}$, respectively. It is clear from Jónsson's lemma that $\mathcal{V}_{2}$ and $\mathcal{V}_{3}$ are minimal varieties.

5.1. Theorem. $\mathcal{V}_{2}$ and $\mathcal{V}_{3}$ are the only minimal subvarieties of $\mathcal{C} \mathcal{I} d \mathcal{R} \mathcal{L}$.

Proof. We show that every non-trivial subvariety $\mathcal{V}$ of $\mathcal{C} \mathcal{I} d \mathcal{R} \mathcal{L}$ contains $\mathbf{C}_{2}$ or $\mathbf{C}_{3}$. According to the well known Magari's theorem, $\mathcal{V}$ contains a (non-trivial) simple algebra $\mathbf{A}$. Indeed, $\left|A^{-}\right|=2$, so $\mathbf{A}$ has the bottom and thus also the top element. We show that $B=\{0, e, 1\}$ is a subalgebra of $\mathbf{A}$ - then it is isomorphic to one of $\mathbf{C}_{2}, \mathbf{C}_{3}$, depending on whether $e=1$ or not. The set $B$ is indeed closed on join, meet and multiplication. In any bounded residuated lattice the equations $x / 0 \approx 1$, $x / e \approx x$ and $1 / x \approx 1$ hold and $0 / 1 \leq e / 1<e$. Hence in a simple CI residuated lattice $0 / 1=e / 1=0$ and we are done.

$\mathcal{V}_{2}$ is known as the variety of generalized Boolean algebras and it is based (relatively to $\mathcal{C} \mathcal{I} \mathcal{R} \mathcal{L})$ by $x \leq e$ and $y /(y / x) \approx x \vee y$. A finite base for the variety $\mathcal{V}_{3}$ can be found in [6] (or computed by the Galatos' algorithm).

In fact, $N$. Galatos proved in [6] that $\mathbf{C}_{2}$ or $\mathbf{C}_{3}$ is a subalgebra of any idempotent residuated lattice $\mathbf{A}$ satisfying $e / x \approx x \backslash e$. If $\mathbf{A}$ is integral, then $\{a, e\}$ is a subalgebra isomorphic to $\mathbf{C}_{2}$ for every $a \neq e$ and if $\mathbf{A}$ is not integral, then $\{e / a, e, e /(e / a)\}$ is a subalgebra isomorphic to $\mathbf{C}_{3}$ for every $a>e$. Consequently, every subvariety of $\mathcal{C} \mathcal{I} d \mathcal{R} \mathcal{L}$ is either integral, or contains $\mathbf{C}_{3}$ (in other words, $\mathbf{C}_{3}$ is a splitting algebra).

Aknowledgement. The paper was written during the author's stay at Vanderbilt University in Nashville, TN, in fall 2003 and 2004. The author is indebted to R. McKenzie for the invitation to Vanderbilt and also to N. Galatos, M. Kozik and C. Tsinakis for their interest, valuable comments and corrections.

\section{REFERENCES}

[1] P. Bahls, J. Cole, N. Galatos, P. Jipsen, C. Tsinakis, Cancellative residuated lattices, Algebra Universalis 50/1 (2003), 83-106.

[2] K. Baker, J. Wang, Definable principal subcongruences, Algebra Universalis 47/2 (2002), 145-151. Zbl 1063.08005

[3] S. Burris, H.P. Sankappanavar, A course in universal algebra, GTM 78, Springer, 1981. Zbl 0478.08001

[4] K. Blount, C. Tsinakis, The structure of residuated lattices, Internat. J. Algebra Comput. 13/4 (2003), 437-461. Zbl 1048.06010

[5] R.P. Dilworth, M. Ward, Residuated lattices, Trans. Amer. Math. Soc. 45 (1939), 335-354. Zbl 0021.10801 
[6] N. Galatos, Minimal varieties of residuated-lattices, Algebra Universalis 52/2-3 (2004), 215239.

[7] N. Galatos, Equational bases for joins of residuated-lattice varieties, Studia Logica 76 (2004), 227-240. Zbl 1068.06007

[8] N. Galatos, Varieties of residuated lattices, PhD Thesis, Vanderbilt University, 2003.

[9] J. Hart, L. Rafter, C. Tsinakis, The structure of commutative residuated lattices, Internat. J. of Algebra Comput. 12 (2002), 509-524. Zbl 1011.06006

[10] P. Jipsen, C. Tsinakis, A survey of residuated lattices, Ordered Algebraic Structures (J. Martinez, ed.), Kluwer Academic Publishers, Dordrecht, 2002, 19-56. Zbl pre02208113

[11] R. McKenzie, Equational bases for lattice theories, Math. Scand. 27 (1970), 24-38. Zbl 0307.08001

[12] R. McKenzie, G. McNulty and W. Taylor, Algebras, Lattices, Varieties, Volume I. Wadsworth \& Brooks/Cole, Monterey, CA, 1987. Zbl 0611.08001

David Stanovský, Charles University in Prague, Czech Republic

STANOVSK@KARLIN.MFF.CUNI.CZ 